Supporting Information

\title{
Total Synthesis of the Proposed Structures of Indole Alkaloids Lyaline and Lyadine
}

\author{
M.-Lluïsa Bennasar, * Tomàs Roca, and Manuel Monerris
}

Laboratory of Organic Chemistry, Faculty of Pharmacy, University of Barcelona, 08028-Barcelona, Spain

bennasar@ub.edu

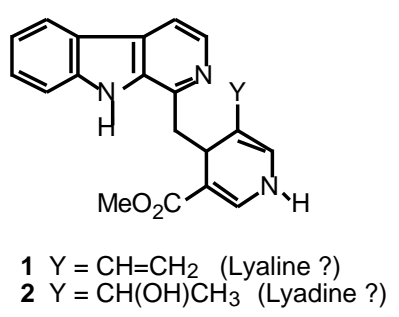

Contents:

- Pages S1 to S5: General experimental protocols and experimental procedures for the preparation of 12a-c, 18a, and 18d.

- Pages S6 to S49: NMR spectra of all compounds.

- Pages S50 and S51: ESI-MS spectra of $\mathbf{1}$ and $\mathbf{2}$. 


\section{General Procedures.}

All nonaqueous reactions were performed under an argon atmosphere. All solvents were dried by standard methods. Reactions courses and product mixtures were routinely monitored by TLC on silica gel (precoated $\mathrm{F}_{254}$ Merck plates). Drying of organic extracts during the workup of reactions was performed over anhydrous $\mathrm{Na}_{2} \mathrm{SO}_{4}$. Evaporation of the solvents was accomplished under reduced pressure with a rotatory evaporator. Flash chromatography was carried out on $\mathrm{SiO}_{2}$ (silica gel 60, SDS, 0.04$0.06 \mathrm{~mm}$ ). Melting points are uncorrected. Chemical shifts of NMR spectra are reported in ppm downfield ( $\delta$ ) from $\mathrm{Me}_{4} \mathrm{Si}$. $N$-methylharman (6) was prepared from commercially available harman following a reported procedure. ${ }^{1}$ Pyridine $8 \mathbf{a}$ was prepared by esterification of commercially available 3,5-pyridinedicarboxylic acid. Pyridines $\mathbf{8 b},{ }^{2} \mathbf{8 c},,^{2,3}$ and $\mathbf{8} \mathbf{d}^{2}$ were prepared following reported procedures.

Organometallic Derivative 9. $n$-BuLi (1.6 M in hexane, $0.35 \mathrm{~mL}, 0.56 \mathrm{mmol}$ ) was slowly added under Ar to a cooled $\left(0^{\circ} \mathrm{C}\right)$ solution of harmane $6(0.10 \mathrm{~g}, 0.51 \mathrm{mmol})$ in dry THF $(10 \mathrm{~mL})$, and the resulting red solution was stirred at $0^{\circ} \mathrm{C}$ for $30 \mathrm{~min}$. After the solution was cooled to $-78^{\circ} \mathrm{C}$, a suspension of $\mathrm{CuCN}$ (46 mg, $0.51 \mathrm{mmol}$ ) in dry THF ( $5 \mathrm{~mL}$ ) was added. The resulting mixture was stirred until the reaction temperature rose to $0^{\circ} \mathrm{C}$, and then cooled again to $-78^{\circ} \mathrm{C}$. $\mathrm{ZnCl}_{2}\left(1 \mathrm{M}\right.$ in $\left.\mathrm{Et}_{2} \mathrm{O}, 0.51 \mathrm{~mL}, 0.51 \mathrm{mmol}\right)$ was added and the mixture was stirred for $10 \mathrm{~min}$. Then was used in the next reaction.

Dimethyl

dihydropyridine-3,5-dicarboxylate (12a). Acetyl chloride $(36 \mu \mathrm{L}, 0.51 \mathrm{mmol})$ was added to a solution of pyridine $\mathbf{8 a}(0.10 \mathrm{~g}, 0.51 \mathrm{mmol})$ in anhydrous THF $(6 \mathrm{~mL})$ cooled at $-10^{\circ} \mathrm{C}$. The resulting mixture was stirred at $-10^{\circ} \mathrm{C}$ for $15 \mathrm{~min}$ and then cooled to $-78^{\circ} \mathrm{C}$. Organometallic $9(0.51 \mathrm{mmol})$ was added by cannula, and the mixture was allowed to slowly warm to $\mathrm{rt}$ for $12 \mathrm{~h}$. The reaction mixture was poured into a $1: 1$ mixture of $20 \% \mathrm{NH}_{4} \mathrm{OH}$ and saturated aqueous $\mathrm{NH}_{4} \mathrm{Cl}(25 \mathrm{~mL})$, and extracted with $\mathrm{CH}_{2} \mathrm{Cl}_{2}(3 \times 30 \mathrm{~mL})$. The organic extracts were concentrated and the resulting residue was chromatographed (95:5 AcOEt-MeOH) to give 1,2-dihydropyridine 14a (20 mg, 9\%) and 1,4-dihydropyridine 12a (90 mg, 41\%). 14a: ${ }^{1} \mathrm{H}$ NMR $\left(\mathrm{CDCl}_{3}, 400 \mathrm{MHz}\right.$, assignments aided by HSQC) $\delta 2.11$ (br s, 3H, COMe), $3.30\left(\mathrm{t}, J=10.7 \mathrm{~Hz}, 1 \mathrm{H}, \mathrm{CH}_{2}\right.$ ), $3.64(\mathrm{~s}, 3 \mathrm{H}, \mathrm{OMe}), 3.65\left(\mathrm{dd}, J=5.2,13 \mathrm{~Hz}, 1 \mathrm{H}, \mathrm{CH}_{2}\right), 3.86(\mathrm{~s}, 3 \mathrm{H}, \mathrm{OMe}), 4.31(\mathrm{~s}, 3 \mathrm{H}$, 
NMe), 6.20 (br s, 1H, pyr 2-H), 7.26 (t, $J=7.2 \mathrm{~Hz}, 1 \mathrm{H}, 6-\mathrm{H}), 7.49$ (d, J = 8.4 Hz, 1H, 8H), 7.59 (t, $J=7.2 \mathrm{~Hz}, 1 \mathrm{H}, 7-\mathrm{H}), 7.67$ (s, 1H, pyr 4-H), 7.84 (d, J = 5.2 Hz, 1H, 4-H), 7.98 (br s, $1 \mathrm{H}$, pyr 6-H), 8.08 (d, $J=7.6 \mathrm{~Hz}, 1 \mathrm{H}, 5-\mathrm{H}), 8.24$ (br s, $1 \mathrm{H}, 3-\mathrm{H}) ;{ }^{13} \mathrm{C}$ NMR $\left(\mathrm{CDCl}_{3}, 100.6 \mathrm{MHz}\right.$, assignments aided by HSQC) $\delta 21.0(\mathrm{COMe}), 32.2(\mathrm{NMe}), 38.3$ $\left(\mathrm{CH}_{2}\right), 50.0$ (pyr C-2), $51.9(\mathrm{OMe}), 52.0(\mathrm{OMe}), 109.7$ (C-8), 113.6 (C-4), 119.6 (C-6), 121.0 (C-4b), 121.4 (C-5), 121.8 (pyr C-5), 128.3 (C-7), 129.5 (C-4a), 130.4 (pyr C-4), 136.2 (C-9a), 137.4 (C-3), 138.9 (pyr C-6), 139.6 (pyr C-3), 142.4 (C-1, C-8a), 165.1 (CO), 165.3 (CO), 169.7 (CO). 12a: ${ }^{1} \mathrm{H}$ NMR $\left(\mathrm{CDCl}_{3}, 300 \mathrm{MHz}\right.$, assignments aided by HSQC and HMBC) $\delta 2.41$ (s, 3H, COMe), 3.2-3.7 (br s, 8H, OMe, $\mathrm{CH}_{2}$ ), $4.30(\mathrm{~s}, 3 \mathrm{H}$, $\mathrm{NMe}), 4.35$ (t, $J=7.5 \mathrm{~Hz}, 1 \mathrm{H}$, pyr 4-H), 7.26 (ddd, $J=1.2,6.9,8.0 \mathrm{~Hz}, 1 \mathrm{H}, 6-\mathrm{H}), 7.4-$ 8.2 (br s, 2H, pyr 2-H and 6-H), 7.48 (d, $J=8.1 \mathrm{~Hz}, 1 \mathrm{H}, 8-\mathrm{H}), 7.59$ (ddd, $J=1.2,6.9$, $8.1 \mathrm{~Hz}, 1 \mathrm{H}, 7-\mathrm{H}), 7.80$ (d, $J=5.1 \mathrm{~Hz}, 1 \mathrm{H}, 4-\mathrm{H}), 8.09$ (d, $J=8.0 \mathrm{~Hz}, 1 \mathrm{H}, 5-\mathrm{H}), 8.22$ (d, $J$ $=5.1 \mathrm{~Hz}, 1 \mathrm{H}, 3-\mathrm{H}) ;{ }^{1} \mathrm{H} \mathrm{NMR}\left(\mathrm{CDCl}_{3}, 300 \mathrm{MHz}, 55{ }^{\circ} \mathrm{C}\right) \delta 3.42(\mathrm{~s}, 6 \mathrm{H}, \mathrm{OMe}), 3.55(\mathrm{~d}, J$ $\left.=7.5 \mathrm{~Hz}, 2 \mathrm{H}, \mathrm{CH}_{2}\right), 7.97$ (s, 2H, pyr 2-H and 6-H); ${ }^{13} \mathrm{C} \mathrm{NMR}\left(\mathrm{CDCl}_{3}, 75.4 \mathrm{MHz}\right.$, assignments aided by HSQC and HMBC) $\delta 21.8$ (COMe), 32.1 (NMe), 33.7 (pyr C-4), $43.4\left(\mathrm{CH}_{2}\right), 51.6(\mathrm{OMe}), 109.5$ (C-8), 113.1 (C-4), 114.2 (pyr C-3 and C-5), 119.4 (C6), 120.9 (C-4b), 121.2 (C-5), 128.1 (C-7), 129.2 (C-4a), 131.1 (pyr C-2 and C-6), 136.1 (C-9a), 137.2 (C-3), 141.4 (C-1), 142.2 (C-8a), 166.4 (CO), 167.2 (CO); HRMS calcd for $\mathrm{C}_{24} \mathrm{H}_{23} \mathrm{~N}_{3} \mathrm{O}_{5} 433.1638$, found 433.1639 .

\section{Methyl 1-Acetyl-5-ethyl-4-[(9-methyl- $\beta$-carbolin-1-yl)methyl]-1,4-}

dihydropyridine-3-carboxylate (12b). Acetyl chloride $(36 \mu \mathrm{L}, 0.51 \mathrm{mmol})$ was added to a solution of pyridine $\mathbf{8 b}(84 \mathrm{mg}, 0.51 \mathrm{mmol})$ in anhydrous THF $(6 \mathrm{~mL})$ cooled at $-25^{\circ} \mathrm{C}$. The resulting mixture was stirred at $-25^{\circ} \mathrm{C}$ for $30 \mathrm{~min}$, cooled to $-78^{\circ} \mathrm{C}$ and then treated with $9(0.51 \mathrm{mmol})$ as above. After the extractive workup and chromatography of the residue (AcOEt) a 4:1:1 mixture of dihydropyridines $\mathbf{1 2 b}, \mathbf{1 4 b}$ and $\mathbf{1 6 b}$ (83 $\mathrm{mg}$, $40 \%$ ) was obtained. An additional chromatography allowed the isolation of pure 12b (2:1 mixture of rotamers): ${ }^{1} \mathrm{H} \mathrm{NMR}\left(\mathrm{CDCl}_{3}, 300 \mathrm{MHz}\right.$, assignment aided by HSQC and HMBC) $\delta 0.85$ and 0.95 (2 br m, $3 \mathrm{H}, \mathrm{CH}_{2} \mathrm{CH}_{3}$ ), 1.4-1.8 (3 br m, $2 \mathrm{H}, \mathrm{CH}_{2} \mathrm{CH}_{3}$ ), 2.34 (s, $3 \mathrm{H}, \mathrm{COMe}$ ), 3.37 and 3.55 (2 br s, 3H, OMe), 3.4-3.7 (br m, 2H, $\mathrm{CH}_{2}$ ), 3.90 (br m, 1H, pyr 4-H), 4.17 (br s, 3H, NMe), 6.40 and 7.0 (2 br s, $1 \mathrm{H}$, pyr 6-H), 7.28 (t, $J=8.2 \mathrm{~Hz}$, $1 \mathrm{H}, 6-\mathrm{H}), 7.48(\mathrm{~d}, J=8.4,1 \mathrm{H}, 8-\mathrm{H}), 7.61(\mathrm{t}, J=7.6 \mathrm{~Hz}, 1 \mathrm{H}, 7-\mathrm{H}), 7.75$ and 8.30 (2 br s, $1 \mathrm{H}$, pyr 2-H), $7.85(\mathrm{~d}, J=5.2 \mathrm{~Hz}, 1 \mathrm{H}, 4-\mathrm{H}), 8.12(\mathrm{~d}, J=8 \mathrm{~Hz}, 1 \mathrm{H}, 5-\mathrm{H}), 8.34(\mathrm{~d}, J=5.2$ 
$\mathrm{Hz}, 1 \mathrm{H}, 3-\mathrm{H}) ;{ }^{13} \mathrm{C} \mathrm{NMR}\left(\mathrm{CDCl}_{3}, 75.4 \mathrm{MHz}\right.$, assignment aided by HSQC and HMBC) $\delta$ $12.0\left(\mathrm{CH}_{2} \mathrm{CH}_{3}\right), 21.7$ and $21.8(\mathrm{COMe}), 25.9\left(\mathrm{CH}_{2} \mathrm{CH}_{3}\right), 32.4(\mathrm{NMe}), 37.4$ (pyr C-4), $42.4\left(\mathrm{CH}_{2}\right), 51.5$ (OMe), 109.5 (C-8), 112.2 (pyr C-3), 113.0 (C-4), 116.6 and 118.2 (pyr C-6), 119.6 (C-6), 121.0 (C-4b), 121.3 (C-5), 127.1 (pyr C-5), 128.2 (C-7), 129.5 (C-4a), 131.9 and 133.3 (pyr C-2), 135.8 (C-9a), 137.9 (C-3), 142.3 (C-1), 142.4 (C-8a), 166.9 (CO), 167.2 (CO); HRMS calcd for $\mathrm{C}_{24} \mathrm{H}_{25} \mathrm{~N}_{3} \mathrm{O}_{3} 403.1896$, found 403.1890.

Methyl

1,5-Diacetyl-4-[(9-methyl- $\beta$-carbolin-1-yl)methyl]-1,4dihydropyridine-3-carboxylate (12c). Acetyl chloride $(36 \mu \mathrm{L}, 0.51 \mathrm{mmol})$ was added to a solution of pyridine $\mathbf{8 c}(91 \mathrm{mg}, 0.51 \mathrm{mmol})$ in anhydrous $\mathrm{CH}_{2} \mathrm{Cl}_{2}(5 \mathrm{~mL})$ cooled at $-10^{\circ} \mathrm{C}$, and the mixture was stirred at $-10^{\circ} \mathrm{C}$ for $5 \mathrm{~min}$. Trimethylsilyl triflate $(92 \mu \mathrm{L}$, $0.51 \mathrm{mmol}$ ) was added and the mixture was stirred at $-10{ }^{\circ} \mathrm{C}$ for $1 \mathrm{~h}$. The resulting pyridinium triflate (11c) was allowed to react as above with $9(0.51 \mathrm{mmol})$. After the extractive workup and chromatography of the residue (96:4 AcOEt-MeOH) 1,4dihydropyridine 12c was obtained: $85 \mathrm{mg}(40 \%) ;{ }^{1} \mathrm{H} \mathrm{NMR}\left(\mathrm{CDCl}_{3}, 300 \mathrm{MHz}\right) \delta 2.26$ (br s, 3H, COMe), 2.44 (s, 3H, COMe), 3.24 (br s, 3H, OMe), 3.3 and 3.5 (2 br m, 2H, $\left.\mathrm{CH}_{2}\right), 4.32(\mathrm{~s}, 3 \mathrm{H}, \mathrm{NMe}), 4.47$ (t, $\left.J=7.2 \mathrm{~Hz}, 1 \mathrm{H}, \mathrm{pyr} 4-\mathrm{H}\right), 7.26(\mathrm{t}, J=7.5 \mathrm{~Hz}, 1 \mathrm{H}, 6-$ H), $7.49(\mathrm{~d}, J=8.1 \mathrm{~Hz}, 1 \mathrm{H}, 8-\mathrm{H}), 7.59$ (t, $J=7.2 \mathrm{~Hz}, 1 \mathrm{H}, 7-\mathrm{H}), 7.80(\mathrm{~d}, J=5.1 \mathrm{~Hz}, 1 \mathrm{H}$, 4-H), 7.85 (br s, 1H, pyr 2-H), 8.08 (d, $J=7.5 \mathrm{~Hz}, 1 \mathrm{H}, 5-\mathrm{H}), 8.18$ (d, $J=5.1 \mathrm{~Hz}, 1 \mathrm{H}, 3-$ $\mathrm{H}), 8.20$ (br s, $1 \mathrm{H}$, pyr 6-H); ${ }^{13} \mathrm{C} \mathrm{NMR}\left(\mathrm{CDCl}_{3}, 75.4 \mathrm{MHz}\right) \delta 21.7$ (COMe), 25.2 (COMe), 32.0 (NMe), 32.3 (pyr C-4), $42.6\left(\mathrm{CH}_{2}\right), 51.4$ (OMe), 109.5 (C-8), 113.1 (C4), 114.7 (pyr C-3), 119.3 (C-6), 120.8 (C-4b), 121.1 (C-5), 123.4 (pyr C-5), 128.1 (C7), 129.2 (C-4a), 131.5 (pyr C-2), 136.0 (C-9a), 136.9 (C-3), 141.4 (C-1), 142.2 (C-8a), 166.0 (CO), 167.4 (CO), 196.2 (CO).

General Procedure for the $N$-deprotection of 12a and 12c. Dihydropyridines 12a,c $(0.1 \mathrm{mmol})$ were added to a solution of $\mathrm{KOH}$ in $\mathrm{MeOH}(1 \%, 3 \mathrm{~mL})$ and stirring was maintained at $\mathrm{rt}$ for $15 \mathrm{~min}$. The reaction mixture was poured into $5 \%$ aqueous $\mathrm{Na}_{2} \mathrm{CO}_{3}(10 \mathrm{~mL})$ and extracted with $\mathrm{CH}_{2} \mathrm{Cl}_{2}(6 \times 20 \mathrm{~mL})$. The organic extracts were dried and concentrated. Pure 18a,c were obtained after flash chromatography. Eluent and yields are given below.

Dimethyl 4-[(9-methyl- $\beta$-carbolin-1-yl)methyl]-1,4-dihydropyridine-3,5dicarboxylate (18a). 98:2 AcOEt-MeOH; yield 85\%; ${ }^{1} \mathrm{H}$ NMR (DMSO-d 6 , $300 \mathrm{MHz}$, 
assignment aided by HSQC and HMBC) $\delta 3.21$ (s, 6H, OMe), 3.24 (d, $J=7.5 \mathrm{~Hz}, 2 \mathrm{H}$, $\mathrm{CH}_{2}$ ), $4.21(\mathrm{t}, J=7.5 \mathrm{~Hz}, 1 \mathrm{H}$, pyr 4-H), 4.25 (s, 3H, NMe), 7.24 (ddd, $J=0.9,7.0,7.8$ $\mathrm{Hz}, 1 \mathrm{H}, 6-\mathrm{H}), 7.30$ (dd, $J=0.9,5.4 \mathrm{~Hz}, 2 \mathrm{H}$, pyr 2-H and 6-H), 7.59 (ddd, $J=1.2,7.0$, $8.4 \mathrm{~Hz}, 1 \mathrm{H}, 7-\mathrm{H}), 7.73$ (d, $J=8.4 \mathrm{~Hz}, 1 \mathrm{H}, 8-\mathrm{H}), 7.92$ (d, $J=5.1 \mathrm{~Hz}, 1 \mathrm{H}, 4-\mathrm{H}), 8.10$ (d, $J$ $=5.1 \mathrm{~Hz}, 1 \mathrm{H}, 3-\mathrm{H}), 8.20(\mathrm{~d}, J=7.8 \mathrm{~Hz}, 1 \mathrm{H}, 5-\mathrm{H}), 9.24(\mathrm{t}, J=5.7 \mathrm{~Hz}, 1 \mathrm{H}, \mathrm{NH}) ;{ }^{13} \mathrm{C}$ NMR (DMSO-d 6 , 75.4 MHz, assignment aided by HSQC and HMBC) $\delta 31.9$ (NMe), 32.5 (pyr C-4), $42.9\left(\mathrm{CH}_{2}\right), 50.7$ (OMe), 104.3 (pyr C-3 and C-5), 110.2 (C-8), 112.8 (C-4), 119.3 (C-6), 120.6 (C-4b), 121.3 (C-5), 128.0 (C-7), 128.1 (C-4a), 135.7 (C-9a), 136.4 (pyr C-2 and C-6), 136.9 (C-3), 141.8 (C-1), 142.3 (C-8a), 167.0 (CO).

\section{Methyl 5-Acetyl-4-[(9-methyl- $\beta$-carbolin-1-yl)methyl]-1,4-dihydropyridine} 3-carboxylate (18c). 9:1 AcOEt-MeOH; yield 80\%; mp 134-135 ${ }^{\circ} \mathrm{C}$; 4:1 mixture of rotamers; ${ }^{1} \mathrm{H}$ NMR (DMSO-d 6 , $300 \mathrm{MHz}$, major rotamer) $\delta 2.03$ (s, 3H, COMe), 3.09 (dd, $\left.J=8.1,12.6 \mathrm{~Hz}, 1 \mathrm{H}, \mathrm{CH}_{2}\right), 3.18\left(\mathrm{dd}, J=6.6,12.6 \mathrm{~Hz}, 1 \mathrm{H}, \mathrm{CH}_{2}\right), 3.13(\mathrm{~s}, 3 \mathrm{H}$, OMe), 4.28 (s, 3H, NMe), 4.33 (dd, $J=8.1,12.6 \mathrm{~Hz}, 1 \mathrm{H}$, pyr 4-H), 7.24 (t, $J=7.8 \mathrm{~Hz}$, 1H, 6-H), 7.28 (d, $J=5.1 \mathrm{~Hz}, 1 \mathrm{H}$, pyr 2-H), 7.48 (d, $J=5.4,1 \mathrm{H}$, pyr 6-H), 7.59 (ddd, $J$ $=0.9,7.8,8.7 \mathrm{~Hz}, 1 \mathrm{H}, 7-\mathrm{H}), 7.73(\mathrm{~d}, J=8.7 \mathrm{~Hz}, 1 \mathrm{H}, 8-\mathrm{H}), 7.90(\mathrm{~d}, J=5.0,1 \mathrm{H}, 4-\mathrm{H})$, $8.08(\mathrm{~d}, J=5.0 \mathrm{~Hz}, 1 \mathrm{H}, 3-\mathrm{H}), 8.19(\mathrm{~d}, J=7.8,1 \mathrm{H}, 5-\mathrm{H}), 9.36(\mathrm{t}, J=5.1 \mathrm{~Hz}, \mathrm{NH}) ;{ }^{13} \mathrm{C}$ NMR (DMSO-d $\mathrm{d}_{6}, 100.6 \mathrm{MHz}$, major rotamer) $\delta 24.8$ (COMe), 31.1 (pyr C-4), 31.9 (NMe), $42.3\left(\mathrm{CH}_{2}\right), 50.6$ (OMe), 105.4 (pyr C-3), 110.3 (C-8), 112.8 (C-4), 115.2 (pyr C-5), 119.3 (C-6), 120.7 (C-4b), 121.3 (C-5), 128.0 (C-7), 128.2 (C-4a), 135.8 (C-9a), 136.0, 136.8 (pyr C-2, C-3), 138.3 (pyr C-6), 141.8 (C-1), 142.5 (C-8a), 166.9 (CO), 194.8 (CO).

\section{References}

1. (a) Karrer, P.; Müller, H. J. Org. Chem. 1957, 22, 1433-1434. (b) Seki, H.; Hashimoto, A.; Hino, T. Chem. Pharm. Bull. 1993, 41, 1169-1172.

2. Bracher, F.; Papke, T. Monatsh. Chem. 1995, 126, 805-809.

3. Bennasar, M.-L.; Zulaica, E.; Roca, T. Alonso, Y.; Monerris, M. Tetrahedron Lett. 2003, 44, 4711-4744. 